\title{
A Study of the Predictability of Tropical Pacific SST in a Coupled Atmosphere-Ocean Model Using Singular Vector Analysis: The Role of the Annual Cycle and the ENSO Cycle*
}

\author{
Ying-Quei Chen And David S. Battisti \\ Department of Atmospheric Sciences, University of Washington, Seattle, Washington \\ T. N. PALMER \\ ECMWF, Shinfield Park, Reading, United Kingdom \\ JOSEPH BARSUGLI \\ CIRES, University of Colorado, Boulder, Colorado \\ E. S. SARACHIK \\ Department of Atmospheric Sciences, University of Washington, Seattle, Washington
}

(Manuscript received 19 June 1995, in final form 23 February 1996)

ABSTRACT

\begin{abstract}
The authors examine the sensitivity of the Battisti coupled atmosphere-ocean model-considered as a forecast model for the El Niño-Southern Oscillation (ENSO) - to perturbations in the sea surface temperature (SST) field applied at the beginning of a model integration. The spatial structures of the fastest growing SST perturbations are determined by singular vector analysis of an approximation to the propagator for the linearized system. Perturbation growth about the following four reference trajectories is considered: (i) the annual cycle, (ii) a freely evolving model ENSO cycle with an annual cycle in the basic state, (iii) the annual mean basic state, and (iv) a freely evolving model ENSO cycle with an annual mean basic state. Singular vectors with optimal growth over periods of 3,6 , and 9 months are computed.

The magnitude of maximum perturbation growth is highly dependent on both the phase of the seasonal cycle and the phase of the ENSO cycle at which the perturbation is applied and on the duration over which perturbations are allowed to evolve. However, the spatial structure of the optimal perturbation is remarkably insensitive to these factors. The structure of the optimal perturbation consists of an east-west dipole spanning the entire tropical Pacific basin superimposed on a north-south dipole in the eastern tropical Pacific. A simple physical interpretation for the optimal pattern is provided. In most cases investigated, there is only one structure that exhibits growth.

Maximum perturbation growth takes place for integrations that include the period June-August, and the minimum growth for integrations that include the period January-April. Maxima in potential growth also occur for forecasts of ENSO onset and decay, while minima occur for forecasts initialized during the beginning of a warm event, after the transition from a warm to a cold event, and continuing through the cold event. The physical processes responsible for the large variation in the amplitude of the optimal perturbation growth are identified. The implications of these results for the predictability of short-term climate in the tropical Pacific are discussed.
\end{abstract}

\section{Introduction}

The El Niño-Southern Oscillation (ENSO) phenomenon is an interannual climate anomaly of large scale

\footnotetext{
* Contribution 331 to the Joint Institute for the Study of the Atmosphere and Ocean.
}

Corresponding author address: Dr. David S. Battisti, Department of Atmospheric Sciences, University of Washington, Box 351640, Seattle, WA 98195-1640.

E-mail: david@atmos.washington.edu that is rooted in the tropical Pacific. ENSO is inherently due to interactions between the atmosphere and the ocean, and it is widely thought that the crucial physics associated with ENSO are contained within the tropical Pacific basin. The tropical aspects of the ENSO phenomenon are summarized in numerous papers, including the seminal paper of Rasmusson and Carpenter (1982). A recent review of the observations is found in Trenberth (1991).

Zebiak and Cane (1985) presented the first coupled atmosphere-ocean model that demonstrated interannual variability similar to the observed ENSO phenomenon. The first forecast of short-term (i.e., months to years) 
climate anomalies was made with this model; the model predicted a development of positive sea surface temperature (SST) anomalies in the tropical Pacific one year in advance of the onset of the 1986-87 warm phase of ENSO (Cane et al. 1986). Since then, a variety of statistical and dynamical coupled models have been developed that possess skill (relative to a persistence forecast) in the prediction of central and eastern Pacific SST one year in advance [see Barnston et al. (1994) and Latif et al. (1994) for up-to-date ENSO prediction reviews]. The forecast skill of an individual model depends on the physics of that model and the method of initialization, among other factors. Dynamical models that are initialized solely by forcing the ocean component of the coupled model with observed winds up until the time of forecast initialization and then diagnostically determining the model atmosphere generally have nowcast (i.e., initial) correlation skills of above 0.8 for the Nino- 3 index ${ }^{1}$ and then maintain useful correlation skills out to at least 1 year (e.g., Cane et al. 1986). More sophisticated initialization procedures can significantly increase the time span over which the model can make skillful forecasts (Chen et al. 1995; Rosati et al. 1997). Statistical models have also demonstrated useful forecast skill out to about 12 months [e.g., cf. rms Nino-3 error in Penland and Sardeshmukh (1995), Fig. 13, and Chen et al. (1995), Fig. 3].

ENSO forecasts are presently being used in many tropical countries with great social and economic benefits (see, e.g., Moura 1994; Battisti and Sarachik 1995). To make rational decisions based on an ENSO forecast, it is important to understand the intrinsic limitations to the forecast accuracy and to identify the sources of possible forecast error. Important and practical questions arise concerning the nature of the observing system required to achieve the most skillful forecasts. Which variables must be monitored? What spatial and temporal resolution is required for each variable to optimize the forecast skill? Is the predictability of the tropical system inherently seasonally dependent? Is the forecast skill of a given model seasonally dependent? How do we discriminate forecast errors due to model imperfections from those that are due to initialization errors?

An important step in understanding the predictability of the tropical Pacific atmosphere-ocean system is to study error growth in a linearized version of an actual prediction model. A traditional starting point is the identification of the most unstable eigenmode of the physical system. This, however, may not be the most useful approach for understanding the predictability of this dynamical system because the linear approximation of the tropical atmosphere-ocean system is a nonnormal dynamical system. A sufficient condition to ensure non-

\footnotetext{
${ }^{1}$ The Nino-3 index refers to the SST anomaly averaged from $5^{\circ} \mathrm{S}$ to $5^{\circ} \mathrm{N}$ and $150^{\circ} \mathrm{W}$ to $90^{\circ} \mathrm{W}$.
}

normality is the asymmetric way in which the atmosphere and ocean are coupled (see section 2). A nonnormal system is also ensured by the annual cycle of the spatially inhomogeneous mean states of both the ocean and the atmosphere. Hence, in order to describe more completely the linear error growth of the system, we need to find a suitable orthogonal basis that describes the finite time linear growth of perturbations to the initial conditions of the system. This basis is provided by the singular vectors of the integral propagator associated with the linearized model (Lorenz 1965; Buizza and Palmer 1995). The propagator is the linear operator that takes perturbations at a given time and "propagates" them to some time in the future; it has also been referred to as the resolvent ${ }^{2}$ or Green function (e.g., Penland and Sardeshmukh 1995). The fastest growing singular vector has also been called an optimal perturbation (e.g., Farrell 1989). The most unstable eigenmode that is ubiquitous to numerous tropical Pacific atmosphere-ocean models is a quasi-stationary eigenmode, which is similar in structure to the observed ENSO and has an $e$-folding time of about 1 year. In this study, we will examine the optimal growth of initial perturbations, which will be seen to be much greater than that associated with the fastest growing eigenmode of the system.

Analysis of the optimal perturbation growth can be used to identify the causes for errors in forecasts, and thus to help answer the five questions noted above (Buizza et al. 1996). In this study, we focus on the perturbation growth that results because of perturbations in the initial conditions (derived by an initialization procedure from an analysis of observations) that are prescribed at the initial time of the model integrations. These uncertainties are understood to be associated with observational (measurement) or initialization errors. In a separate study, we use this model to perform retrospective ENSO forecasts and address the issue of whether the forecast errors can be attributed to errors in the initial conditions, or whether they must be due to errors in the model physics.

In particular, we focus our efforts here on evaluating the sensitivity of the final state SST field to small perturbations in the initial state of the SST in the model. Appropriately, an accurate prediction of the final state SST field has been the Holy Grail of short-term climate forecasts in the Tropics because the low-frequency (e.g., seasonal to interannual) atmospheric circulation anomalies of global proportions are strongly related to tropical Pacific SST anomalies. The fact that we consider initial perturbations only to the model SST (and the associated wind field; see model description below) is justified not only by its intrinsic interest, but by our desire to answer in a later study whether errors in SST

\footnotetext{
${ }^{2}$ More on the theory of the non-self-adjoint systems and their predictability can be found in Farrell (1990).
} 
alone could be responsible for the errors in actual forecasts of ENSO, even if the initial state of the thermocline were known exactly. This is not to deny the potential importance of thermocline perturbations to a full understanding of ENSO predictability.

The model used in this study is Battisti's (1988) version of the Zebiak and Cane (1987) model and is described in section 2. A brief review of the theory of singular vector growth and a description of the method used to obtain an approximate linear version of the full model are presented in section 3. We present in section 4 the optimal growth of perturbations as a function of the phase of the seasonal cycle and the ENSO cycle from which the integration is initiated. We discuss and interpret the results in the context of the model physics in section 5. Finally, in section 6, we present our conclusions based on the major findings of this work. Preliminary results of singular vector calculations using this model were reported in Palmer et al. (1994).

\section{The coupled atmosphere-ocean model}

The coupled atmosphere-ocean model used in this study is described in Battisti (1988, hereafter B88), which follows very closely the coupled model of Zebiak and Cane (1987, hereafter ZC). This coupled model consists of an ocean whose evolution is treated prognostically, coupled to an atmosphere whose state is determined diagnostically at each time step ( $\Delta t=10$ days; Zebiak 1986). Both the ocean and the atmosphere are anomaly models constructed about a monthly varying annual cycle. The ocean is forced by the anomalous surface wind stress and the atmosphere is forced by a parameterized anomalous latent heat release. The latent heat release is a function of the climatological convergence of the wind field and the total SST. Both of these forcing terms are nonlinear. The ocean model domain is a rectangular basin spanning $30^{\circ} \mathrm{S}-30^{\circ} \mathrm{N}$ and $124^{\circ} \mathrm{E}-$ $80^{\circ} \mathrm{W}$; the atmosphere and ocean dynamics are modeled on an equatorial $\beta$ plane.

The ocean model consists of an upper layer overlying a deep motionless layer. The upper layer contains within it a fixed depth surface mixed layer used for thermodynamic calculations. The upper-layer depth and currents are governed by linear shallow-water wave dynamics. SST is calculated separately and does not directly affect the ocean dynamics. SST is determined through nonlinear temperature advection by surface currents, through upwelling into the surface layer, and through heat fluxes to the atmosphere. These terms are calculated as follows: the surface currents are driven by the wind stress and retarded by Rayleigh friction, and the upwelling is prescribed in terms of the divergence of the surface-layer currents. The mean state of the ocean surface-layer temperature (i.e., SST) about which the anomaly model is constructed is that given by Rasmusson and Carpenter (1982; hereafter RC). The mean surface-layer currents are calculated by forcing the mod- el with the RC annual cycle winds until a cyclic steady state is reached.

The atmosphere model is the linear two-layer Gill (1980) model of a thermally forced tropical atmosphere. The forcing of the atmosphere depends on the total atmospheric convergence and the SST and is calculated iteratively (Zebiak 1986)-the so-called convergence feedback effect. The atmosphere is treated diagnostically and only changes on timescales associated with the SST changes. The equations that comprise the coupled model are found in appendix A of Battisti (1988).

One-way forcing experiments with the atmosphere and ocean models separately indicate that each component of the coupled model produces a reasonable simulation of the observed interannual variability in the region of the oceanic equatorial waveguide. The evidence for this is as follows. The ocean model, when forced by observed wind stress anomalies, has been shown to provide a good simulation of the observed SST and sea level anomalies from 1961 to 1992 (Mantua and Battisti 1994). When the atmospheric model is forced by either the RC composite ENSO SST anomalies or the 1982-83 Pacific SST anomalies, the surface wind anomalies near the equator are similar to the observed anomalies (Zebiak 1986). There are, however, significant differences between the simulated and observed anomalies in the wind field away from the equator at all longitudes (e.g., cf. Gill 1980; Gill and Rasmusson 1984).

When the atmosphere and ocean models are coupled, the model supports interannual variability that has much in common with observed ENSO events. Specifically, the spatial structure of the model ENSO is of basin scale, with greatest SST anomalies in the eastern and central Pacific; the model events are locked to the annual cycle, with simulated and observed warm events generally peaking in December-January; the variance in SST is predominantly at the interannual timescale, with substantially less variability at subannual timescales (Mantua and Battisti 1995). The simulated ENSO events recur every 3 or 4 years-much more regularly than observed ENSO events. Finally, the physics of the interannual variability displayed by this coupled atmosphere-ocean model is well described by the delayed oscillator mechanism (Battisti and Hirst 1989).

\section{Methods}

\section{a. Theory of linear perturbation growth: Singular vector analysis}

We write the evolution equations for the perturbation state vector $\mathbf{X}$ (X may include surface currents, thermocline displacement, and SST perturbations) for the linearized coupled atmosphere-ocean system in matrix form,

$$
\frac{\partial \mathbf{X}}{\partial t}=\mathbf{L X} .
$$


Here, the matrix $\mathbf{L}$ is a nonnormal operator ${ }^{3}$ for reasons stated in section 1 . Hence the eigenvalues of $L$ do not bound the maximum transient growth of perturbations, as they would for a normal operator. Assuming both the dynamical system equations and the initial state are known exactly, the future state can be determined by integrating Eq. (1) with the appropriate initial conditions. In practice, the initial conditions are only known to finite accuracy. The solution to Eq. (1) for the perturbation state vector $\mathbf{X}$ at time $t+\tau$ is given by

$$
\mathbf{X}(t+\tau)=\mathbf{R}(t, \tau) \mathbf{X}(t) .
$$

Here, $\mathbf{R}$ is the propagator. When the basic state trajectory is time independent, then

$$
\mathbf{R}(t, \tau)=\exp \left(\int_{t}^{t+\tau} \mathbf{L} d t\right) .
$$

We choose as our measure of the perturbation vector the norm based on the standard inner product applied to the state vector, $\|\mathbf{X}\|^{2} \equiv\langle\mathbf{X}(t), \mathbf{X}(t)\rangle=\mathbf{X} * \mathbf{W} \mathbf{X}$, where $\mathbf{W}$ is a diagonal weighting matrix, which we take to be the identity matrix for the purposes of this discussion. (In principle, $\mathbf{W}$ can be any positive definite matrix, and can be used to implement norms based on perturbation energy or other physically based quantities.)

The amplification of the perturbation vector over time $\tau$ is, therefore,

$$
\begin{aligned}
A & =\frac{\|\mathbf{X}(t+\tau)\|}{\|\mathbf{X}(t)\|}=\frac{\langle\mathbf{X}(t+\tau), \mathbf{X}(t+\tau)\rangle^{1 / 2}}{\langle\mathbf{X}(t), \mathbf{X}(t)\rangle^{1 / 2}}, \\
& =\frac{\langle\mathbf{R} \mathbf{X}(t), \mathbf{R} \mathbf{X}(t)\rangle^{1 / 2}}{\langle\mathbf{X}(t), \mathbf{X}(t)\rangle^{1 / 2}}=\frac{\langle\mathbf{X}(t), \mathbf{R} * \mathbf{R} \mathbf{X}(t)\rangle^{1 / 2}}{\langle\mathbf{X}(t), \mathbf{X}(t)\rangle^{1 / 2}} .
\end{aligned}
$$

The operator $\mathbf{R} * \mathbf{R}$ is normal, so the eigenvalues are positive real numbers. The set of eigenvectors of $\mathbf{R} * \mathbf{R}$ form an orthonormal basis, which can be used to describe arbitrary perturbations to the model state vector.

Here, $\mathbf{R}$ can be written in terms of singular value decomposition (e.g., Strang 1988):

$$
\mathbf{R}=\mathbf{F} \boldsymbol{\Lambda} \mathbf{E}^{*} \text {, }
$$

where $\boldsymbol{\Lambda}$ is a real, positive, diagonal matrix, and $\mathbf{E}, \mathbf{F}$ are orthonormal (unitary) matrices. The diagonal entries of $\boldsymbol{\Lambda}$ are referred to as singular values, and the columns of $\mathbf{E}$ and $\mathbf{F}$ are referred to as right and left singular vectors, respectively. From Eq. (5), we see that the singular values of $\mathbf{R}$ are the square roots of the eigenvalues of $\mathbf{R} * \mathbf{R}$ :

$$
\mathbf{R} * \mathbf{R}=\mathbf{E} \boldsymbol{\Lambda}^{2} \mathbf{E}^{*} .
$$
by

Hence, from Eq. (4), the maximum value of $A$ is given

\footnotetext{
${ }^{3}$ That is, $\mathbf{L} * \mathbf{L} \neq \mathbf{L} \mathbf{L}^{*}$, where the superscript asterisk indicates the complex-conjugate transpose.
}

$$
\frac{\left\langle\mathbf{E}_{1}(t), \lambda_{1}^{2} \mathbf{E}_{1}(t)\right\rangle^{1 / 2}}{\left\langle\mathbf{E}_{1}(t), \mathbf{E}_{1}(t)\right\rangle^{1 / 2}}=\lambda_{1},
$$

where $\lambda_{1}$, the largest singular value of $\mathbf{R}$, therefore bounds the perturbation growth. The corresponding right singular vector, $\mathbf{E}_{1}$, is the pattern of the initial perturbation that leads to the greatest perturbation growth. When we refer to singular vector without qualification we will mean the right singular vector or equivalently the initial pattern of the optimal perturbation. Examination of Eq. (5) shows that $\mathbf{R}(t, \tau) \mathbf{E}_{1}=\lambda_{1} \mathbf{F}_{1}$. Hence, $\lambda_{1} \mathbf{F}_{1}$, derived by applying the propagator to the initial pattern, is the corresponding final pattern, and that it has amplitude $\lambda_{1}$. The above discussion also holds when $\mathbf{R}$ is restricted to act on a subset of the complete state vector.

\section{b. Calculation of the linear propagator $\mathbf{R}$}

Before we proceed with the singular vector analysis, we must first obtain the propagator for the linearized system. The propagator describes the evolution of perturbations about a reference trajectory. Rather than code a linearized version of the numerical model, we choose a simpler, approximate method of obtaining the propagator directly from the full coupled model. We follow the procedure outlined below:

1) The full model is first integrated from the initial time $t$ to some later time, $t+\tau$. This control integration represents a reference trajectory. In this study, we take $\tau$ to be 3,6 , or 9 months.

2) The integration is then repeated, placing a small positive perturbation of amplitude $\epsilon$ in one element of the state vector (i.e., one unit in one variable at one grid point and zero otherwise) at the initial time $t$. The perturbation at the final time $(t+\tau)$ that is due to the initial perturbation applied at time $(t)$ is given by the difference between the perturbed and the reference states at time $t+\tau$. The initial and final perturbation vectors are recorded as one column vector in the initial and final perturbation matrices $\mathbf{X}_{i}$ and $\mathbf{X}_{f}$, respectively, [see Eq. (2)].

3) Step two is repeated, placing perturbations at each grid point of the state vector. Record the initial and final perturbation state vectors in matrices $\mathbf{X}_{i}$ and $\mathbf{X}_{f}$ accordingly.

This procedure (1-3) generates the relation

$$
\mathbf{R}(t, \tau) \mathbf{X}_{i}=\mathbf{X}_{f},
$$

and was proposed in the meteorological literature as early as Lorenz (1965). Hence, the approximation to the linear propagator for the numerical model is $\mathbf{R}(t, \tau)=$ $\mathbf{X}_{f} \mathbf{X}_{i}^{-1}$. As stated above, if we interpret the initial perturbation as the error or uncertainty at the initial time, the maximum possible error growth is the first (largest) singular value of the propagator, $\mathbf{R}$. The initial and final patterns that accomplish this error growth are the right and left singular vectors of $\mathbf{R}$. 


\section{c. The choice of the norm}

We examine in this study the maximum growth of perturbations in the SST field of the coupled atmosphere-ocean model described in section 2 that results from uncertainties in the initial conditions in the SST field. We focus on identifying the spatial structure of the fastest growing perturbations in tropical SST $\left(12^{\circ} \mathrm{S}-\right.$ $12^{\circ} \mathrm{N}$ ) because the low-frequency tropical Pacific atmospheric circulation (thus the tropical climate) is intimately tied to the SST distribution in the equatorial tropical Pacific. Furthermore, climate variability over the North Pacific sector has been shown to be statistically related to the SST distribution in the tropical Pacific (e.g., Ropelewski and Halpert 1987).

The choice of norm must be consistent with the probability distribution of errors in the initial state (Molteni et al. 1995). In general this distribution is poorly known. Previous studies of the stability of geophysical flows have focused on maximizing either the final-state enstrophy (e.g., Farrell 1990) or total perturbation energy (e.g., Buizza and Palmer 1995; Moore and Kleeman 1996). Virtually all models used to forecast the state of the tropical Pacific climate system require at initialization time both the SST and the upper-ocean density structure (or pycnocline position). Numerous models also impose the state of the global atmosphere in their initialization. We will limit this study to focus on how perturbations in SST at the initialization time evolve to produce the optimal amplification in SST at the end state. Hence, the state vector (see section $3 b$ ) contains only the SST component, though $\mathbf{R}$ contains the full model physics for propagating the initial SST anomalies to the final state.

We apply two tests to determine whether the propagator generated by the method outlined above is a good approximation to the actual linear propagator. First, we follow steps 1-3 using various values for $\epsilon$, ranging from $0.01^{\circ}$ to $2^{\circ} \mathrm{C}$, positive or negative. For all cases investigated the largest singular value determined by using positive perturbations and negative perturbations agreed to within 5\%. The associated singular vector determined by these two approaches are also in excellent quantitative agreement. All results presented in this paper use $\epsilon=0.5^{\circ} \mathrm{C}$.

In the second test, the full numerical coupled model was initialized with the optimal perturbation (i.e., the first singular vector) obtained from the singular-vector analysis, and the coupled model was integrated to obtain the final perturbation pattern. Both the final state pattern and the perturbation growth are compared to those obtained using the propagator $\mathbf{R}$. For the 6-month integrations that we have performed, typical pattern correlation for the final state resulting from the nonlinear numerical model integration and that resulting from the linear propagator is about 0.99 , and the difference between the amplification factor estimated by the linear

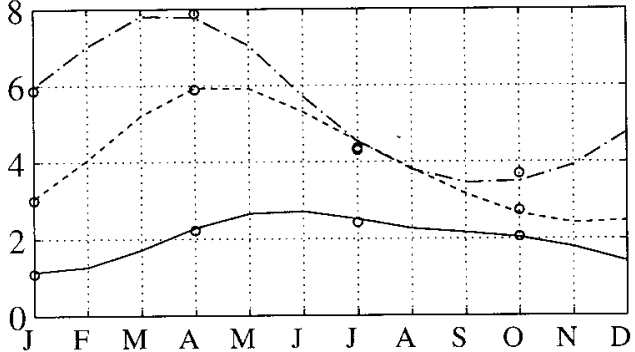

FIG. 1. The optimal growth of SST ( $\lambda_{1}$, the first singular value of R) as a function of the phase of the annual cycle, for 3-month (solid), 6-month (dashed), and 9-month (dash-dot) integrations about the prescribed climatological annual cycle reference trajectory (see section 4a). For example, the maximum perturbation growth over a 6-month period starting in April and ending in October is 5.9. The initial amplitude of SST in perturbed integrations (see section 3b, step 2) is $\epsilon=0.5^{\circ} \mathrm{C}$. Indicated by circles are the first singular values with $\epsilon=-0.5^{\circ} \mathrm{C}$.

propagator and that obtained from the nonlinear model is typically less than $10 \%$.

\section{Idealized singular-vector growth experiments \\ a. Climatological annual cycle reference trajectory}

We examine how the initial perturbations applied in different months of the annual cycle evolve over a chosen duration of $\tau=3,6$, and 9 months. For this set of experiments the reference trajectory is exactly the prescribed climatological annual cycle. The initial and final state perturbations are deviations about the annual cycle. For the parameters used in this study, the coupled model annual cycle is unstable to infinitesimal perturbations (Battisti and Hirst 1989), and in the presence of any initial noise a model ENSO cycle is eventually set up. However, the spatial structure of singular vectors for the timescales investigated here tends to be insensitive to whether or not the normal modes of the system are unstable, and the analysis presented here should carry over to the case in which the model is in a stable regime.

The growth of the first singular vector (i.e., the value of $\lambda_{1}$ ) is strongly dependent on the calendar month in which the perturbation is applied and on the duration of the integration. The first singular values for 3-, 6-, and 9-month integrations are plotted in Fig. 1 as a function of the month in which the perturbed integration is started. The maximum growth for the 3-month integrations ranges from a factor of 2.7 for the integration that starts in June to 1.1 for the integration that starts in January. In most cases, $\lambda_{1}$ increases as the duration of integration increases. For the 6-month integration, $\lambda_{1}$ ranges from 5.9 for the April start, decreasing to 2.4 for the November start; for the 9-month integration, $\lambda_{1}$ ranges from 7.8 for the March start to 3.5 for the September start. The results point to a period of greater sensitivity during boreal summer because the periods of maximum growth over 3,6 , and 9 months are all centered on this season. 
(a) JAN Initial Condition : SV $=3.06$

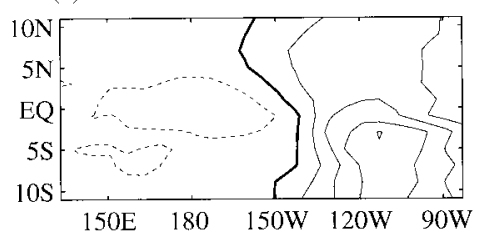

(b) APR Initial Condition : SV $=5.94$

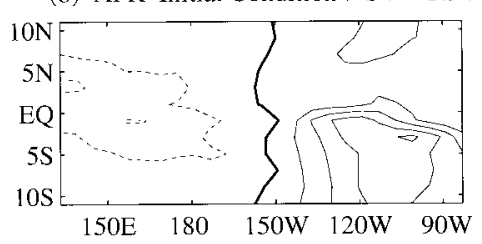

(c) JUL Initial Condition: SV $=4.54$

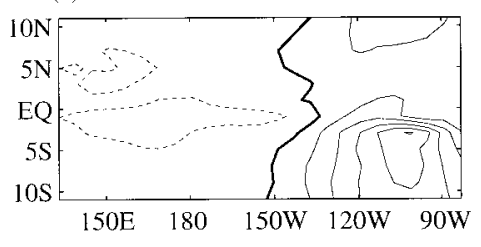

(d) OCT Initial Condition: $\mathrm{SV}=2.66$

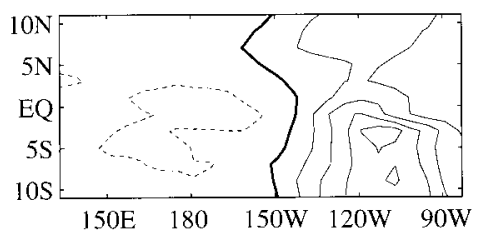

6-Month Later : JUL

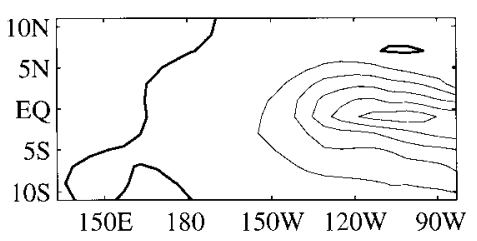

6-Month Later : OCT

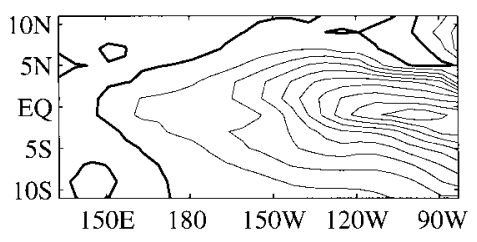

6-Month Later : JAN

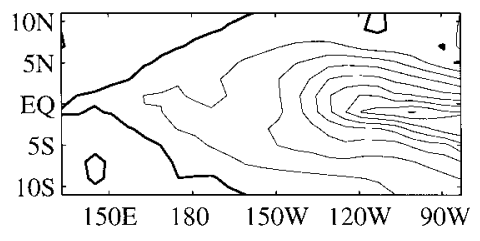

6-Month Later : APR

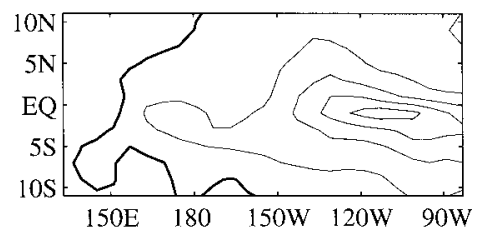

FIG. 2. (a) The optimal SST perturbation applied in January (left) that produces the maximum amplitude in SST six months later in July (right). Panels (b), (c), and (d) are for optimal perturbations applied in April, July, and October, respectively. The contour interval for the initial (final) perturbations in the left (right) column is $0.04^{\circ} \mathrm{C}\left(0.12^{\circ} \mathrm{C}\right)$. For each case, $\lambda_{1}$ is noted above the left panel.

The reader will note the circles plotted in Fig. 1, which indicate the first singular values associated with the propagators that are obtained in exactly the same manner as is outlined in section $3 \mathrm{~b}$ but with negative initial perturbations $\left(\epsilon=-0.5^{\circ} \mathrm{C}\right)$ applied to the model in step 2 . The growth $\left(\lambda_{1}\right)$, initial, and final state patterns of the optimal perturbation that are calculated from the propagator obtained by applying positive/negative perturbations to the model are in excellent quantitative agreement. The results in Fig. 1 are robust to the amplitude of initial perturbation that is applied to the model to construct the linear propagator (see section 3c), suggesting we have obtained a good approximation to the linear propagator $\mathbf{R}$.

In contrast to the sensitivity of the singular values, both the initial and final patterns are not sensitive to the month in which the initial perturbation is applied nor to the duration over which the perturbation is allowed to grow. Plotted in Fig. 2 are the optimal initial patterns, $\mathbf{E}_{1}$ (left-hand column, recall $\left\|\mathbf{E}_{1}\right\|=1$ ), and the corresponding final patterns, $\lambda_{1} \mathbf{F}_{1}$ (that result from applying the propagator to the optimal initial pattern), for 6-month integrations, starting from January, April, July, and October, respectively. The initial pattern consists of an east-west dipole spanning the entire tropical Pacific basin superimposed upon a north-south dipole in the eastern tropical Pacific. The final pattern resembles the mature model ENSO mode. The patterns for the 3-, 9-, and 12-month integrations are very similar to those for the 6-month integrations and are not shown.

The second singular vectors indicate decay for all of the monthly starts and for all duration of integrations that were examined $(3,6,9$, and 12 months). Note that as the integration time increases, the optimal initial pattern explains a higher proportion of the total information in $\mathbf{R} * \mathbf{R}$. The trace of the matrix $\mathbf{R} * \mathbf{R}$ is proportional to the increase in variance of perturbations, which are initially uniformly distributed [Lorenz (1965); also our Eq. (8), with $\mathbf{X}_{i}$ set to the identity matrix]. The first singular vector accounts for $18 \%-60 \%$ of the total variance of $\mathbf{R} * \mathbf{R}$ for the 3-month integrations; $88 \%-97 \%$ and $97 \%-$ 99\% for 6- and 9-month integrations, respectively. 

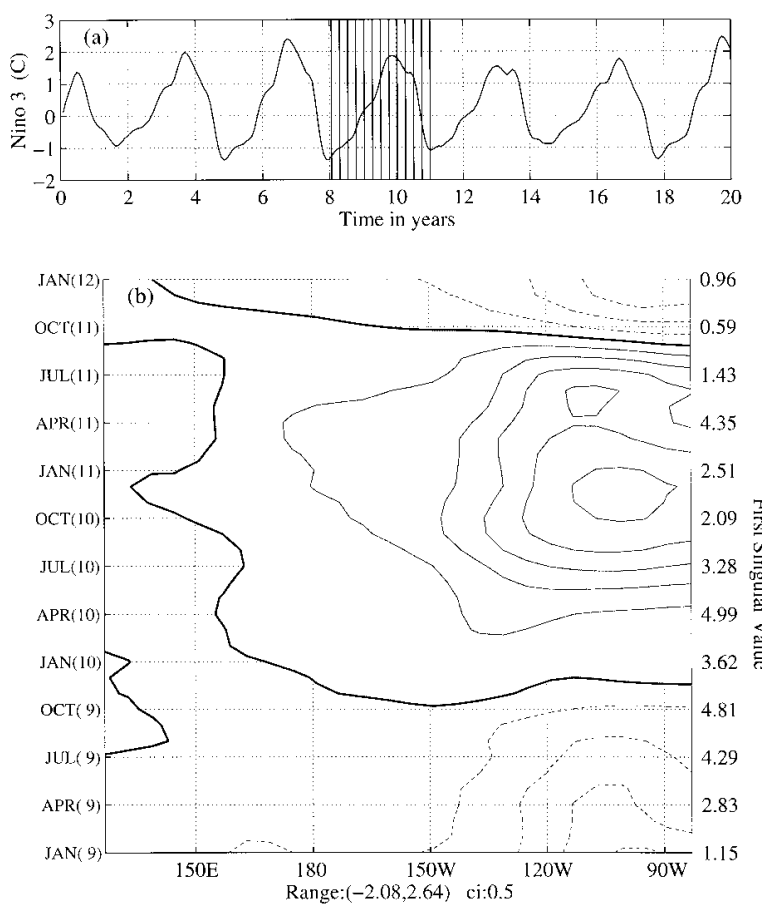

FIG. 3. (a) The $20-y r$ time series of the Nino-3 index from the control integration of the coupled atmosphere-ocean model. (b) Time-longitude section of SST from the (arbitrarily chosen) fourth model ENSO cycle (SST is averaged from $2^{\circ} \mathrm{S}$ to $2^{\circ} \mathrm{N}$; contour interval is $0.5^{\circ} \mathrm{C}$ ). Positive (negative) contours are in solid (dashed) lines Zero contours are in heavy lines. The first singular values for calculations starting with different months/phases [indicated by vertical lines in (a)] for 6-month integrations are written on the right ordinate.

\section{b. ENSO with annual cycle in basic state as reference trajectory}

We next examine how the interannual variability in the coupled atmosphere-ocean model affects the growth of initial perturbations in the SST. The freely evolving coupled model with a seasonal cycle in its basic state (SST, ocean currents, upwelling, thermocline depth, and surface wind) supports interannual variability that resembles the observed ENSO, with model ENSO events occurring 3 or 4 years apart (Battisti 1988). Figure 3a shows a 20-yr time series of the control-run Nino-3 index. The extrema of model SST anomalies ranges from $2.6^{\circ} \mathrm{C}$ in the warm phase to $-2^{\circ} \mathrm{C}$ in the cold phase. We arbitrarily select the ENSO cycle occurring during model years 9, 10, and 11 for further analysis. Figure $3 \mathrm{~b}$ shows the time-longitude section of the equatorial SST anomalies averaged from $2^{\circ} \mathrm{S}$ to $2^{\circ} \mathrm{N}$ for this typical model ENSO cycle.

To determine the optimal perturbations for selected phases from the control integration of the coupled model, restart files were written every month during the control integration. These restart files contain the initial conditions from which we add small perturbations and calculate the singular vectors, as described in section $3 \mathrm{~b}$. The fundamental difference between these calcu-

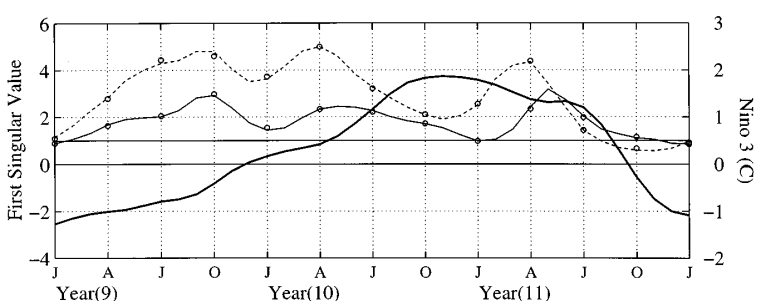

FIG. 4. The first singular value $\left(\lambda_{1}\right)$ for 3-month (solid) and 6-month (dashed) integrations performed on a freely evolving model ENSO reference trajectory, which includes both the interannual variability and the prescribed annual cycle (see section $4 \mathrm{~b}, \epsilon=0.5^{\circ} \mathrm{C}$ ). The Nino-3 index (the fourth cycle in Fig. 3a) is plotted in heavy solid line as a reference for the state of the coupled atmosphere-ocean model (units denoted on the right ordinate). The circles indicate the first singular values with $\epsilon=-0.5^{\circ} \mathrm{C}$.

lations and those presented in section $4 \mathrm{a}$ is summarized as follows. The reference trajectory in the experiments of section $4 \mathrm{a}$ is the climatological annual cycle. The reference trajectory (the control integration) for the experiments described in this section is a freely evolving model ENSO cycle with the annual cycle in the basic state (see, e.g., Fig. 3a).

Singular values and singular vectors are calculated for 3- and 6-month integrations starting from each month of the selected ENSO cycle. The first integration starts in January of year 9, denoted Jan(9), and the last integration starts in $\operatorname{Jan}(12)$. The values of the 6-month optimal perturbation growth $\left(\lambda_{1}\right)$ for every 3 months are shown in Fig. 3b. The 3- and 6-month optimal growths are plotted in Fig. 4 along with the Nino-3 index.

The perturbation growth depends on both the phase of the annual cycle and the phase of the model ENSO cycle. For example, for the 3 -month integrations, growth is largest for $\operatorname{Oct}(9)\left(\lambda_{1}=2.9\right)$, just prior to the onset of the model ENSO event, and for May(11) $\left(\lambda_{1}=3.2\right)$, just prior to the breakdown of the model ENSO event. The growth over 6 months generally exceeds the growth over 3 months, with large growth $\left(\lambda_{1}>3\right)$ throughout the ENSO cycle, except at peak of the warm event $[\operatorname{Aug}(10)-\operatorname{Jan}(11)]$ and during the demise of the warm event [Jun(11)], and continuing through the first half of the model cold event [Apr(12), not shown] where the 6-month optimal perturbation actually decays $\left(\lambda_{1}<1\right)$.

Examples of the patterns of the initial and final state SST that are associated with the optimal growth of SST for the 6-month integrations of the freely evolving coupled model are displayed in Fig. 5. While the maximum perturbation growth is sensitive to the phase of the ENSO cycle in which the perturbation is applied, and hence to the state of the coupled system, both the initial and final patterns of the optimal are insensitive to the initial conditions (cf. Figs. 2 and 5).

\section{c. Annual mean basic-state reference trajectory}

To isolate the effect of the ENSO cycle from that of the basic-state annual cycle, we performed experiments 
(a) Oct $(9$ ) Initial Condition : SV $=4.81$

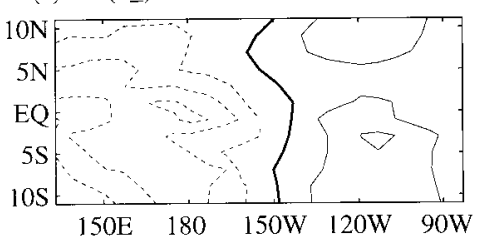

(b) Apr(10) Initial Condition : SV $=4.99$

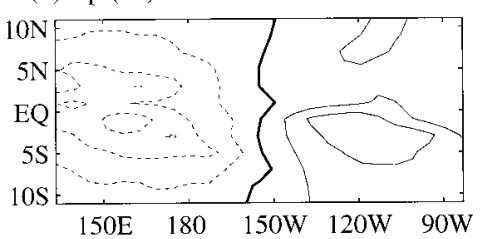

(c) Oct(10) Initial Condition : SV $=2.09$

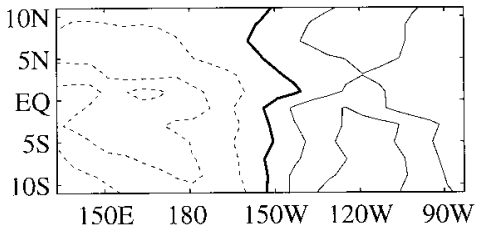

(d) Jul(11) Initial Condition: SV $=1.43$

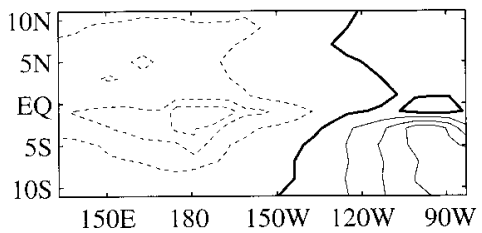

6-Month Later

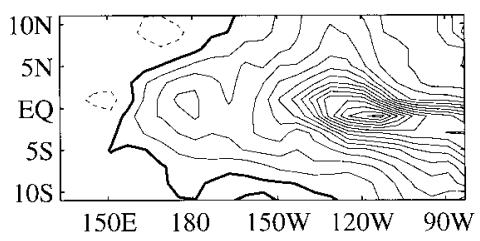

6-Month Later

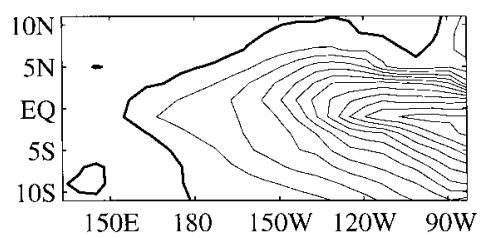

6-Month Later
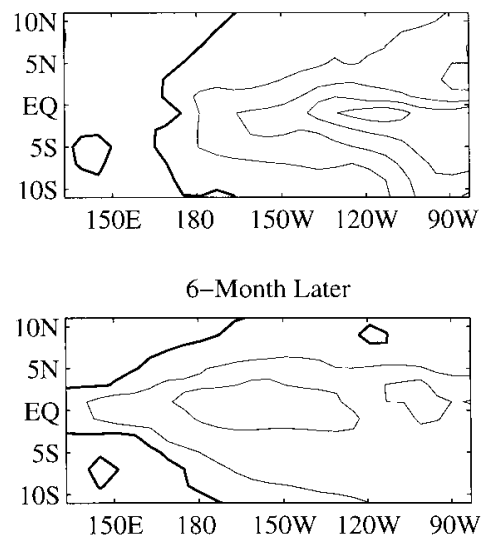

FIG. 5. As in Fig. 2, but for 6-month integrations performed on a freely evolving model ENSO reference trajectory, which includes both the interannual variability and the prescribed annual cycle. Plotted in left and right columns are the initial and final patterns of the optimal perturbation for integrations starting at a specific phase of the model ENSO cycle: (a) prior to event onset [Oct(9)]; (b) during a warming phase [Apr(10)]; (c) at the peak of a warm event [Oct(10)]; (d) during the decaying phase [Jul(11)]. Contour interval in the left (right) column is $0.04^{\circ} \mathrm{C}\left(0.08^{\circ} \mathrm{C}\right)$.

in which the annual cycle is eliminated from the model. To accomplish this, we prescribe a basic state for the coupled model that is the annual mean climatological SST, ocean surface currents, upwelling, thermocline depth, and surface wind stress. In this third experiment, we perturb the model about the time-invariant trajectory that is exactly the annual mean climatology. The 3-month and 6-month optimals have first singular values

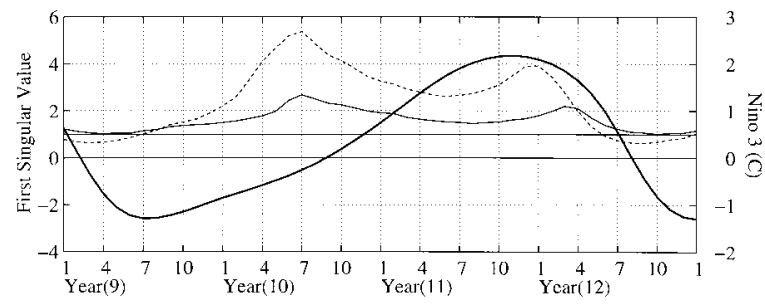

FIG. 6. As in Fig. 4 but using for the annual mean basic state. of 2.0 and 4.2, respectively. The spatial pattern of the optimal perturbation in SST is very similar to those shown in Figs. 2 and 5 and is not shown.

\section{d. ENSO with annual mean basic state as reference trajectory}

The model with the annual mean basic state (see section 4c) supports a freely evolving ENSO cycle with a period of 3.45 years and with extrema in the Nino-3 index of $2.1^{\circ} \mathrm{C}$ and $-1.4^{\circ} \mathrm{C}$. The propagator and singular vectors were obtained following the same procedure as described in section $3 \mathrm{~b}$ but using this freely evolving ENSO with no annual cycle as the reference state. The maximum perturbation growth as a function of the phase of the ENSO cycle in which the integration is begun is displayed in Fig. 6. The maximum growth for both the 3-month $\left(\lambda_{1}=2.7\right)$ and 6-month $\left(\lambda_{1}=5.4\right)$ integrations is achieved just prior to the transition from a cold to 
warm state (month 7 of year 10); a secondary peak in growth in the 6-month integration occurs just prior to the beginning of the demise of the warm event (the first month of year 12). For the 3-month integrations, growth is small just prior to the peak phase of the warm event, and growth is extraordinarily small throughout the first half of the cold phase. For the 6-month integrations, no growing singular vectors are found throughout the first half of the cold phase.

The spatial pattern of the optimal perturbation bears a strong likeness to that for the annual cycle reference trajectory (Fig. 2), and for the "ENSO with annual cycle" reference trajectory (Fig. 5). Overall, Figs. 1, 4, and 6 indicate that the interannual variability generally reduces the sensitivity of the system to SST perturbations except for the periods immediately preceding the onset and demise of the model ENSO warm event. These results are discussed further in sections 5 and 6 .

\section{Discussion}

Three general phenomena stand out in the above experiments. The spatial patterns of the optimal initial perturbation in SST and the resulting final perturbation show almost no dependence on the phase of the annual cycle, the phase of the model ENSO cycle, and the time over which the perturbation is allowed to grow. The final perturbation pattern is very similar to the first empirical orthogonal function (EOF) of the SST from the freely evolving coupled model (i.e., the model ENSO mode; see Mantua and Battisti 1995). The perturbation growth is sensitive to the annual cycle and to the ENSO cycle. Here we present an interpretation of these phenomena based on the physics and dynamics of the model.

The robust initial SST perturbation pattern has a simple physical interpretation. The anomalous atmospheric circulation that would result from the optimal initial SST perturbations shown in Figs. 2 and 5 includes a relaxation of the southeasterly trade winds along the equator in the central-eastern basin along the oceanic equatorial waveguide $\left(5^{\circ} \mathrm{S}-5^{\circ} \mathrm{N}\right)$. To illustrate this point, we apply the 6-month optimal perturbation for the climatological October condition (i.e., Fig. 2d) in the coupled model and integrate forward. The sign of the perturbation is chosen so that it evolves into an ENSO warm event. Figure 7 plots the immediate response in various fields at day 10 (left column) and month 3 (right column) of the perturbed integration (perturbations about the climatological annual cycle). Because of the spatially varying climatological winds, the initial SST perturbations (Fig. 2d) lead to maximum zonal and meridional wind stress anomalies along the equator in the central and eastern Pacific (see Fig. 7b and a close-up in Fig. 8). The anomalous westerly wind stress in the central $\mathrm{Pa}-$ cific leads to warming of the ocean in the central and eastern Pacific (Fig. 7a) by (i) depressing the pycnocline both locally and in the eastern Pacific (Fig. 7e), thus reducing the entrainment rate (Fig. 7d); and (ii) anomalous zonal warm advection in the surface layer (Fig. 7c).

The north-south asymmetry across the equator in the eastern Pacific is a robust feature of the first singular vector and for the polarity chosen above immediately results in a northerly wind anomaly across the equator (Fig. 8). This represents a relaxation of the mean southerly wind stress in the equatorial eastern Pacific $\left(\sim 5^{\circ} \mathrm{S}-\right.$ $5^{\circ} \mathrm{N}$ ) that acts to warm the eastern near-equatorial ocean by decreasing the upwelling of cold water into the surface layer. At first this result was surprising, though in retrospect it could have been anticipated from the basin adjustment study of Philander and Pacanowski (1981) and from the sensitivity studies reported in Battisti (1988, section 4c).

The annual cycle is seen to modulate the growth of the optimal perturbation about a reference trajectory when the ENSO cycle is included (cf. Figs. 1, 4, and $6)$. In general, the presence of the ENSO cycle suppresses the sensitivity of the system to initial perturbations compared to the sensitivity about the annual cycle alone. To make this point clear, we compare the mean 6-month optimal growth in the four experiments. When ENSO cycle is added to the annual mean (Fig. 6 , section $4 d$ ), the average of the first singular values throughout a complete cycle is 2.6-much less than 4.2, the first singular value for the annual mean reference trajectory (section $4 \mathrm{c}$ ). The only times when the first singular value is greater than 4.2 are in months 5-9 of year 10, just prior to the onset of an ENSO warm event. At this time the system is close to its climatological mean state in SST and thermocline depth in the eastern Pacific, and is therefore most sensitive to perturbations. The same conclusion holds when the annual cycle is included. The average of the 12 first singular values of the annual cycle reference trajectory is 4.0 (Fig. 1, section $4 \mathrm{a})$. When the ENSO cycle is added to the annual cycle (Fig. 4, section 4b), the average of the first singular values throughout a complete cycle is 2.9-again, less than 4.0.

The ENSO cycle alone is most sensitive to SST perturbations during the transition from cold to warm Nino-3 SST (and to a lesser extent to the transition from warm to cold). In Fig. 9, we compare the first singular values for the annual-cycle reference trajectory (also displayed in Fig. 1) with the first singular values for the freely evolving model ENSO trajectory (also displayed in Fig. 4) for 3-month (Fig. 9a) and 6-month (Fig. 9b) integrations. The only time during the ENSO cycle that the amplification of the 6-month optimal perturbation growth is enhanced compared to the amplification about the climatological reference trajectory is for perturbations that are applied just prior to the transition from a cold to warm phase of ENSO $[\operatorname{Aug}(9)$ to $\mathrm{Feb}(10)$ in Fig. 9b]. However, for the 3-month integrations, the ENSO cycle enhances the perturbation growth prior to both the transition from cold to warm conditions 
(a) Day_10 SST : ci=0.04C

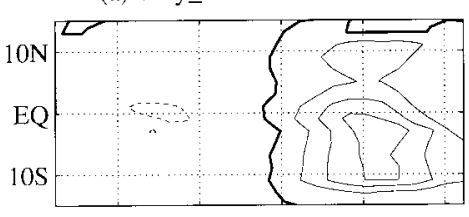

(b) Day_10 tau : ci=0.4m2/s2

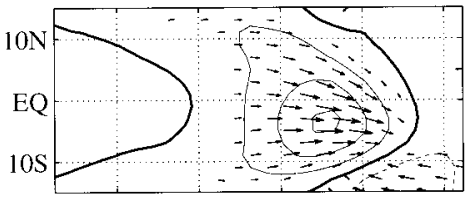

(c) Day_10 usfc: ci=0.01m/s

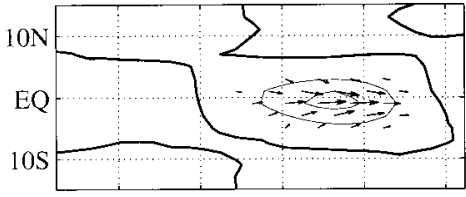

(d) Day_10 w : ci $=0.04 \mathrm{~m} /$ day

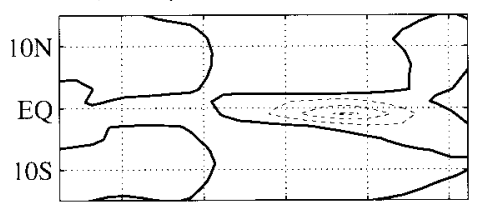

(e) Day_10 h : ci=0.1m

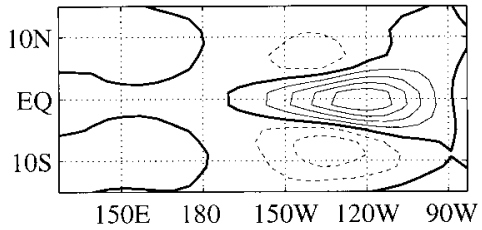

Month 3 SST : ci $=0.08 \mathrm{C}$

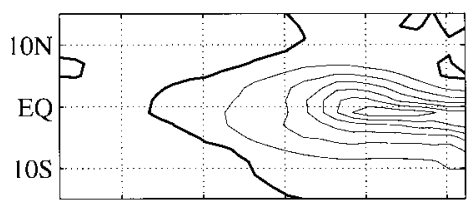

Month_3 tau : ci=0.6m2/s2

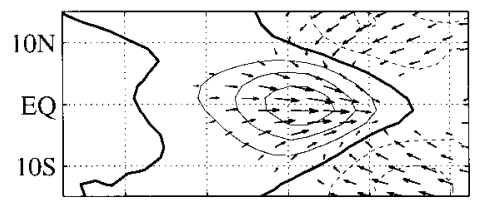

Month_3 usfc : ci $=0.02 \mathrm{~m} / \mathrm{s}$
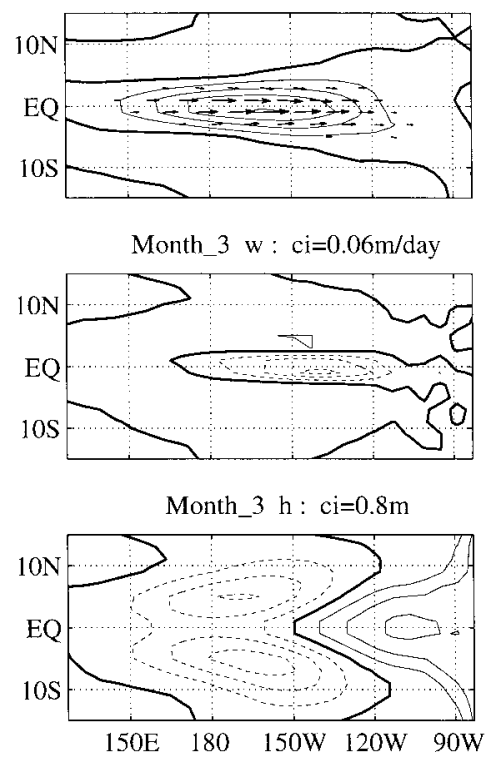

FIG. 7. Coupled model response $\left(15^{\circ} \mathrm{S}-15^{\circ} \mathrm{N}\right)$ in various fields after the 6-month optimal perturbation in SST (first singular vector, Fig. 2d) associated with the climatological October condition is applied: (a) SST, (b) surface wind stress, (c) surface current, (d) upwelling, and (e) the thermocline depth perturbations. Left column is for day 10 response and right column is model response at 3 months. Contour interval is noted on the top of each panel.

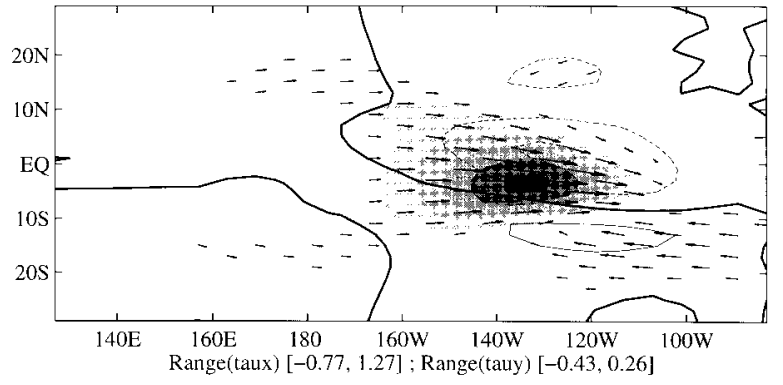

FIG. 8. The immediate response of the atmosphere model in surface wind stress anomaly resulting from perturbing the SST with the 6-month optimal (shown in Fig. 2d) for the climatological October condition. Positive zonal wind stress anomalies are shaded dark (shading level is $0.2 \mathrm{~m}^{2} \mathrm{~s}^{-2}$ starting $+0.2 \mathrm{~m}^{2} \mathrm{~s}^{-2}$ ), and meridional wind stress anomalies are contoured (contour interval is $0.2 \mathrm{~m}^{2} \mathrm{~s}^{-2}$ ).
[Aug(9)-Apr(10)] and the transition from warm to cold conditions [Apr(11)-Jun(11) in Fig. 9a].

The fact that singular values of the nonlinear model trajectory are smaller than the corresponding singular values of the climatological basic state (about which the nonlinear model is constructed), appears to be consistent with the notion that the effect of nonlinearity causes the model to adjust away from the unstable climatological basic state, and toward a more stable part of phase space (S. Zebiak 1995, personal communication to TNP). For example, the farther the system is from climatology, the more the nonlinear terms reduce the local instability in the eastern Pacific. Hence, one might expect on general grounds that the singular vectors of the model ENSO basic state are smaller than those of the climatological 

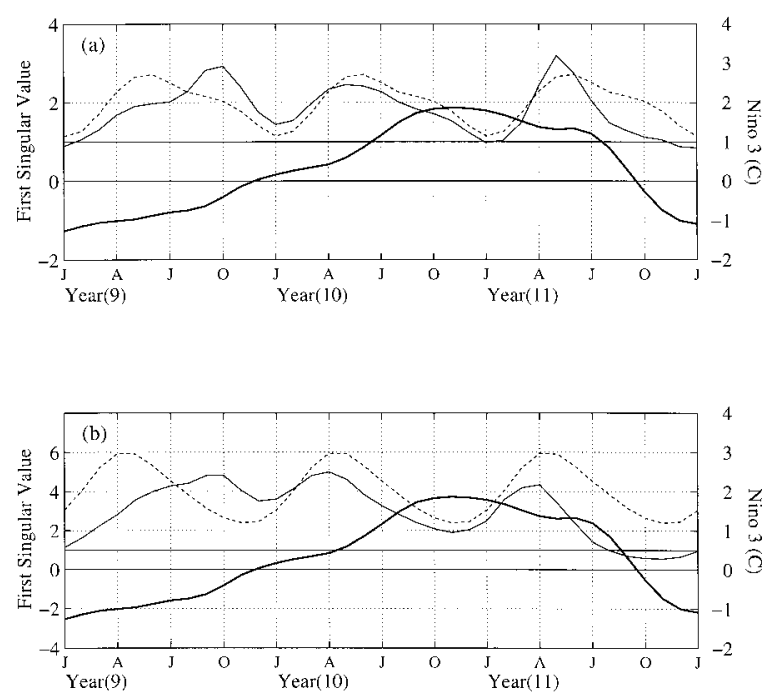

FIG. 9. The first singular value $\left(\lambda_{1}\right)$ about the climatological annual cycle reference trajectory (from Fig. 1, repeated cyclically, dashed line) are superposed on the maximum perturbation growth based on the freely evolving model ENSO trajectory (from Fig. 4, solid line): (a) 3-month and (b) 6-month integrations. The heavy line is the Nino-3 index.

basic state. Indeed, the dominant reason for the general suppression of growth by inclusion of the ENSO cycle is that throughout most of the warm and cold phases of the ENSO cycle the thermocline along the equator is far from its climatological position. Initial perturbations in the SST field will generate anomalies in the wind stress field that, in turn, will force thermocline perturbations. In the warm phase the thermocline is positioned deeper and the upwelling is weaker than in climatological mean fields at that same time of year. Hence, the same perturbation applied to the model while it is in a warm phase will lead to less growth in the final state than for perturbations added to a climatological mean state. Similarly, perturbations applied to the model while it is in the early and middle stages of a cold phase grow less rapidly than perturbations applied to climatological mean conditions because the thermocline is sufficiently shallow. In the cold phase of ENSO, the large downward excursions of thermocline are required to change the temperature of the water that is being entrained into the mixed layer and the upward displacement is limited by the turbulent mixing at the surface (see the appendix). That the model is more sensitive during ENSO transition periods than during warm or cold events is explained by this mechanism as well. That the ENSO transitions can show greater sensitivity than the corresponding basic state alone is not well understood, but most likely involves an additional effect due to the inertia present during ENSO transitions in the form of oceanic Rossby and Kelvin waves.

To understand which physical processes contribute significantly to singular vector growth, we performed several experiments using modified versions of the cou-

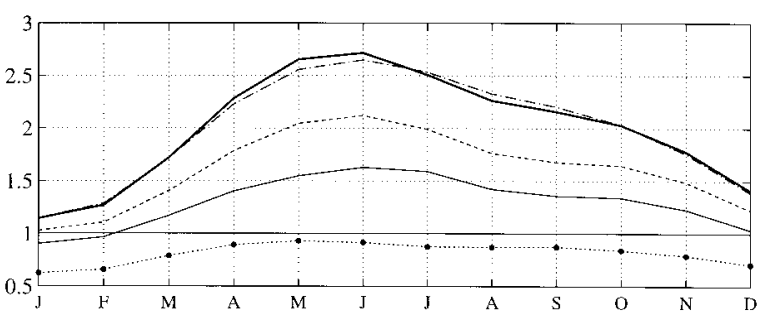

Fig. 10. The first singular value $\left(\lambda_{1}\right)$ for 3-month integrations performed on the annual cycle reference trajectory: standard full model (heavy solid line), no horizontal advection (solid), $\bar{v}_{\text {sfc }}=0$ (dashed), $\bar{u}_{\mathrm{sfc}}=0$ (dash-dot), and no subsurface temperature anomalies, $T_{s}=$ 0 (dotted).

pled model. Here we will report on experiments in which we remove individual terms in the SST equation and then calculate the singular values for the annual cycle reference trajectory. These singular values are compared with those from the full model (section 4a).

The first set of experiments illustrates the importance of thermocline displacements (and the consequent anomalous subsurface temperatures) to perturbation growth by comparing the average of the singular values obtained for the 3-month optimals about the annual cycle with and without this process included in the SST equation. The results from the full model (Fig. 10 heavy solid line, repeated from Fig. 1) indicate that the average of the 12 singular values for the 3-month optimal growth (i.e., the average growth over the annual cycle) is 2.0. However, when thermocline anomalies are not allowed to affect SST, ${ }^{4}$ the annual average of the singular value for the 3-month optimal perturbation is 0.8 , and there are no months that support growing perturbations $\left(\lambda_{1}\right.$ $<1$ for all months; see Fig. 10 dotted line).

The net horizontal temperature advection in the surface layer also affects the amplitude of the singular values, though to a lesser extent than the thermocline displacements. For example, when all horizontal advection terms are removed from the SST equation, the analysis indicates that integrations beginning in all months except January and February support growing perturbations (Fig. 10 solid line); the annual average of the first singular values for the 3-month optimal for the model without horizontal advection is 1.3. Among the four components of horizontal advection:

$$
\begin{array}{r}
\text { (i) } u_{\mathrm{sfc}} \frac{\partial}{\partial x}(\bar{T}+T) \text {, (ii) } v_{\mathrm{sfc}} \frac{\partial}{\partial y}(\bar{T}+T), \text { (iii) } \bar{u}_{\mathrm{sfc}} \frac{\partial T}{\partial x}, \\
\text { and (iv) } \bar{v}_{\mathrm{sfc}} \frac{\partial T}{\partial y},
\end{array}
$$

the most important term for determining growth is the meridional advection by the mean flow [term (iv), Fig. 10 dashed line]. The least important term is the zonal

\footnotetext{
${ }^{4}$ This is accomplished by setting $T_{s}=0$, as discussed in the appendix.
} 
advection by the mean flow [term (iii), Fig. 10 dashdot line]: neglect of this term leads to 3-month singular values, which differ from those of the full model by less than $4 \%$.

The initial perturbation in SST evolves into a final SST perturbation pattern that closely resembles the most unstable eigenmode of the coupled model (Battisti and Hirst 1989). Since the pattern of the final SST perturbation is largely independent of the duration for growth over a time period of 3 months or more, it is temptingbut incorrect - to interpret the final perturbation pattern as the most unstable eigenmode of the coupled system. This pattern is achieved in only about three months, while the timescale associated with setting up the eigenmode is approximately determined by the adjustment time of the equatorial waveguide, which is about 9 months. Nevertheless, the rotation in phase space of the dominant singular vector from an initial direction, which is essentially orthogonal to the fastest growing normal mode, toward a final direction, which is almost parallel to the fastest growing normal mode, is a general characteristic of optimal perturbation growth when there is an unstable normal mode (see Buizza and Palmer 1995).

For perturbations that grow over a time that is short compared to the basin adjustment time, the growth is best thought of as being due to a local atmosphereocean instability that is optimally realized by the initial perturbation previously discussed; the localization is due by the asymmetries of the climatological mean state (Battisti and Hirst 1989). Inspection of Fig. 1 indicates that maximum growth is realized in the boreal summer months: the maximum growth occurs in the 3-month integration spanning June-July-August, and in the 6-month integration spanning April-October. The period of weakest growth occurs in late boreal winter and boreal spring: the weakest growth over a 3-month interval is from January through April, and over a 6-month interval, from November through next May. The explanation for the seasonal dependence lies in the seasonality of the strengths of the mean upwelling and the mean meridional current $\bar{v}_{\text {sfc }}$. Both are largely determined by the wind-driven Ekman transport and are maximum in amplitude in the late boreal summer. Hence, the processes that most amplify the optimal initial SST perturbation are also responsible for the strong seasonality in the amplification of the optimal perturbation. This seasonal dependence in the 3- and 6-month integrations is consistent with Battisti's (1988) results concerning the seasonality in the local stability properties of the mean state of the atmosphere-ocean system in the eastern equatorial Pacific.

\section{Conclusions}

We have examined the sensitivity of the Battisti (1988) coupled atmosphere-ocean model to perturbations in the sea surface temperature (SST) field applied at the beginning of model integrations. The spatial struc- tures of the fastest growing SST perturbations are determined by singular vector analysis of an approximation to the propagator for the linearized system. Four reference trajectories are analyzed: (i) the annual cycle, (ii) a freely evolving model ENSO cycle with the annual cycle in the basic state, (iii) the annual mean climatology (i.e., seasonless), and (iv) a freely evolving model ENSO cycle with an annual mean basic state. Singular vectors with optimal growth for these idealized scenarios over periods of 3,6, and 9 months are computed. Parallel analysis of the integrations with a stable version of the numerical model indicates all of the major results presented in this paper are independent of whether or not the model supports an unstable eigenmode.

The magnitude of maximum growth is shown to be highly dependent upon both the phase of the annual cycle and the phase of the ENSO cycle at which the perturbation is applied and upon the duration that perturbations are allowed to evolve. However, the spatial structure of the optimal perturbation is remarkably insensitive to these factors. The structure of the optimal perturbation consists of an east-west dipole in the entire tropical Pacific basin superimposed on a north-south dipole spanning the eastern tropical Pacific and has a simple physical interpretation. The growth is facilitated by two processes (illustrated here for perturbations that lead to warming). (i) Weakened trades associated with the optimal SST perturbation lead to a deepening of the thermocline along the equator in the eastern Pacific and warming of the surface by changing the temperature of the water being entrained into the mixed layer. The weakened meridional wind stress along the equator in the eastern Pacific reduces the strength of upwelling and also enhances warming. (ii) Meridional advection of these anomalies also contributes significantly to the perturbation growth.

The maximum growth takes place for integrations that include the period June-August and the minimum growth for integrations that include the period JanuaryApril. The seasonal dependence of the optimal perturbation growth is consistent with the seasonality in the local stability of the coupled system in the central and eastern Pacific basin.

Maxima in singular vector growth also occur at specific phases of the model ENSO cycle, with the largest amplifications occurring prior to the onset and decay of the ENSO warm phases, while minima occur for integrations starting at the peak of the warm event, after the transition from a warm to a cold phase and continuing into the cold event. Nonetheless, the overall effect of the ENSO cycle is to reduce the optimal growth compared to growth on the annual-cycle-alone reference trajectory.

It is notable that the spatial structure of the optimal perturbation in SST is of large zonal scale $(\sim 15000$ $\mathrm{km})$. This is consistent with an analysis of the atmospheric response as a function of the zonal scale of the atmospheric heating anomaly. In this model the heating 
is roughly proportional to the SST anomaly. To achieve the greatest dynamical response in the equatorial waveguide (so that the ocean dynamics will be instrumental in affecting the SST later) requires forcing by a zonal wind stress anomaly within the equatorial waveguide (Clarke 1982, personal communication). It can be shown that for the Gill-type models used in this and other coupled models for ENSO, the zonal wind stress anomaly in the equatorial waveguide resulting from a given amplitude of heating (SST) anomaly is maximized when the heating has a zonal scale that is roughly the longitudinal extent of the Pacific Ocean basin, approximately $15000 \mathrm{~km}$. The combination of maximum amplitude response and a large zonal extent ensures that the dynamical response in the ocean will be optimally realized when the initial perturbation in the SST is large scale.

The wind stress (e.g., Fig. 8) and SST patterns (Fig. 2 and Fig. 5) could certainly correspond to plausible errors in the initial conditions for a coupled atmosphereocean model forecast if they were obtained from standard analyses of global observations. In practice, with limited observational data such errors are often strongly determined by the ability of the atmospheric model used in the analysis to simulate the strength of the divergent circulations over the Pacific basin, this in turn being related to the quality of the physical parameterizations in the atmospheric model. The ubiquitous initial singular vector we have found, which is associated with a weakening in the SST gradient across the Pacific basin, is consistent with an erroneously weak Walker circulation in the atmospheric initial state. On the basis of this argument, an initial state with excessively strong divergent circulations would be equally likely.

Chen et al. (1995) as well as previous versions of the ZC model do not use SST observations in their initialization scheme. Instead they initialize their forecast model using analyzed winds. If these winds are incorrect in the sense discussed above, then their initialization scheme could lead to initial SST errors, which project strongly onto our first singular vector.

In most cases examined the optimally growing pattern is also the only growing pattern. Because the final pattern is similar to the model ENSO, this means that incorrect forecasts of the magnitude, the timing, or even the sign of an ENSO event could be compensated for by small corrections in the SST field at the time of the forecast. If the (measurement) error in SST projects strongly onto the first growing singular vector and is not removed from the initial conditions, large errors in the forecast will result. For example, if the error in the initial SST field had the pattern displayed in Fig. 5a with a maximum amplitude of $0.1^{\circ} \mathrm{C}$, it would grow in 6 months to have a rather large error, with maximum amplitude of $0.9^{\circ} \mathrm{C}$ in SST and a similar pattern to the observed ENSO phenomenon. On the other hand, because the error growth is dominated by only one growing pattern, forecast errors that do not project onto the final pattern cannot be compensated for by small corrections in initial SST alone. Therefore, much of the observed SST variability outside of the equatorial waveguide cannot be better predicted by a better SST initialization using this model.

Finally, we note that Moore and Kleeman (1996; hereafter MK) have calculated the optimal growth patterns and rates for a coupled atmosphere-ocean of the tropical Pacific and find somewhat different results from what we have presented for the Battisti (1988) model. One important difference between these two studies is in the choice of variables that are initially perturbed and how the growth is measured. We have chosen to optimize the amplitude of the final state SST perturbation due to perturbation (uncertainty) in the SST field at the beginning of the integration. In contrast, MK optimize the total perturbation energy in the coupled system due to an initial perturbation in the total perturbation energy. MK find that the initial SST perturbations that make up part of their optimal perturbation energy is characterized by very small zonal scales, which quickly damp to reveal the large-scale structure. In addition, a large portion of the optimal error growth in the MK model is mainly attributed to perturbations in the ocean dynamics and less so to the accompanying SST perturbations.

We did not choose to perform calculations to optimize the total perturbation energy using our model, but one might anticipate the initial optimal patterns will be sensitive to the choice of the norm that is being optimized (which is specific to the problem being analyzed) (Farrell 1989). We note, however, that the initial optimal perturbations in SST obtained by Thompson (1997) for the Hirst (1988) model suggest the optimal SST patterns are of large-scale for a variety of norms, and important contributions to the amplification of SST are associated with the initial perturbations in SST, and less so in the perturbations of the dynamics.

The most likely explanation for the differences in the structure of the SST pattern associated with the optimal perturbation for the two models involves the differences in model physics. There are large differences in the governing equations for the two coupled models. In particular, MK make several assumptions that simplify the air-sea coupling and the SST equation used in the present study. Differences in the prescription of the atmospheric heating rate corresponding to a given SST field, and hence differences in the coupling between the ocean and atmosphere, may also play a role. It is well known that fundamental differences in the nature of the interannual variability simulated by freely evolving coupled models arise because of seemingly subtle changes in the ocean thermodynamics (e.g., Hirst 1986). Thus, we expect that even subtle differences in the ocean thermodynamic equation in the two models may greatly affect the optimal growth. Indeed, we find qualitative differences in the optimal growth when various simplifications are made to the ocean thermodynamic equation.

Blumenthal (1991) has examined the optimal pertur- 
bation growth for the Zebiak and Cane (1987) model by first constructing a low-order Markov model that is a best fit to the output of the full numerical ZebiakCane coupled model, and then calculating the adjoint of the normal modes of this Markov model. The adjoint vectors are closely related to our singular vectors in that they provide an initial condition that efficiently excites the model ENSO mode, though they are not necessarily "optimal" for growth over a finite time interval. His choice of norm gives equal weight to thermocline displacement, wind stress, and SST perturbations. Several properties of the optimal growth associated with this Markov model are also common to our numerical model: (i) there is one adjoint that grows much faster than all other singular vectors; (ii) the largest (smallest) 9-month growth is associated with perturbations applied in spring (fall); (iii) the SST component of the adjoint is of large scale (though with a structure that is different from that shown in this study); (iv) the adjoint evolves into a final state whose SST pattern is very similar to the ENSO footprint.

Penland and Sardeshmukh (1995), using a linear stochastic ENSO model derived from observations, also find only one growing singular vector. The large-scale aspects of this singular vector possess some similarity to the singular vectors in the present study, particularly in the north-south and east-west SST gradients in the eastern tropical Pacific. However, their pattern includes a warm anomaly just east of the dateline and a banded structure in the Northern Hemisphere subtropics, which are not seen in the optimal perturbations of our model. Their pattern also has large amplitude in the Indian Ocean, a region which was not included in our study.

The concordance between the results from the Markov model (Blumenthal 1991), the statistical model (Penland and Sardeshmukh 1995), and dynamical models (MK and this study) gives further credence to the idea that predictions of ENSO are usually sensitive to only one pattern of initial SST error.

Acknowledgments. This work was supported by grants from the NOAA Office of Global Programs to DSB and ESS and to the Hayes Center. JJB was supported by a grant from the Climate Dynamics Division of the National Science Foundation (ATM-9313383). We thank the reviewers whose comments make this paper a large improvement over the first manuscript. The work described in this paper began as a superproblem for the 1993 Friday Harbor summer school, organized by Professor Peter Rhines. Our thanks, therefore, to Professor Rhines for facilitating the early stages of this work.

\section{APPENDIX}

\section{Parameterization of the Subsurface Temperature Anomaly}

Perturbations of the model thermocline depth $h$ about the mean state $\bar{h}(x, y)$ give rise to subsurface (at the bottom of the surface mixed layer) temperature anomalies $\left(T_{s}\right)$. In the presence of upwelling $(w)$, these anomalies will cause a change in sea surface temperature anomalies $(T)$ through

$$
w \frac{T-T_{s}}{H_{1}},
$$

where $H_{1}$ is the depth of the mixed layer.

The parameterization for the subsurface temperature anomaly in the B88 model is a modified version of that used by $\mathrm{ZC}$ :

$$
T_{s}=\Theta(h)\{\tanh [\lambda(\bar{h}+1.5|h|)]-\tanh (\lambda h)\},
$$

where $\Theta$ and $\lambda$ are constants, whose values depend only on the sign of the thermocline depth perturbation $h$ (see B88, appendix A). The parameterization (A2) is developed in ZC and is based on an empirical fit of the temperature at the base of the mixed layer $\left(H_{1}\right.$ is taken to be $50 \mathrm{~m}$ ) and the position of the thermocline (see also Seager et al. 1988).

The greatest sensitivity in the heating tendency (A1) will result where thermocline perturbations $(h)$ yield greatest perturbations to $T_{s}$ in (A2), which is where the thermocline is at its climatological annual mean position $(h=0)$.

In the equatorial Pacific, observations indicate there is a lower limit to the subsurface temperature: it rarely drops below $15^{\circ} \mathrm{C}$. A physical justification for the parameterization (A2) for the temperature of the water that is being entrained to the mixed layer is as follows. When the thermocline is very close to the sea surface, the stratification at the base of the mixed layer is large. Hence, the subsurface temperature $T_{s}$ becomes nonlinearly saturated as it becomes more difficult to change the temperature of the water that is entrained into the mixed layer.

\section{REFERENCES}

Barnston, A. G., and Coauthors, 1994: Long-lead seasonal forecasts -Where do we stand? Bull. Amer. Meteor. Soc., 75, 2097-2114.

Battisti, D. S., 1988: The dynamics and thermodynamics of a warming event in a coupled tropical atmosphere-ocean model. J. Atmos. Sci., 45, 2889-2919.

—, and A. C. Hirst, 1989: Interannual variability in the tropical atmosphere-ocean system: Influence of the basic state, ocean geometry, and nonlinearity. J. Atmos. Sci., 46, 1687-1712.

_ , and E. S. Sarachik, 1995: Understanding and predicting ENSO. IUGG Contributions in Oceanography. Eos, Trans. Amer. Geophys. Union, 33, 1367-1376.

Blumenthal, M. B., 1991: Predictability of a coupled atmosphereocean model. J. Climate, 4, 766-784.

Buizza, R., and T. N. Palmer, 1995: The singular-vector structure of the atmospheric general circulation. J. Atmos. Sci., 52, 14341456.

— , R. Gelaro, F. Molteni, and T. N. Palmer, 1996: The impact of increased resolution on predictability studies with singular vectors. Quart. J. Roy. Meteor. Soc. 122, in press.

Cane, M. A., S. E. Zebiak, and S. C. Dolan, 1986: Experimental forecasts of El Niño. Nature, 321, 827-832.

Chen, D., S. E. Zebiak, A. J. Busalacchi, and M. A. Cane, 1995: An 
improved procedure for El Niño forecasting: Implications for predictability. Science, 269, 1699-1702.

Gill, A. E., 1980: Some simple solutions for heat-induced tropical circulation. Quart. J. Roy. Meteor. Soc., 106, 447-462.

— , and E. M. Rasmusson, 1984: The 1982-83 climate anomaly in the equatorial eastern Pacific. Nature, 306, 229-234.

Farrell, B. F., 1989: Optimal excitation of baroclinic waves. J. Atmos. Sci., 46, 1193-1206.

- 1990: Small error dynamics and the predictability of atmospheric flows. J. Atmos. Sci., 47, 2409-2416.

Hirst, A. C., 1986: Unstable and damped equatorial modes in simple coupled ocean-atmosphere models. J. Atmos. Sci., 43, 606-630.

- 1988: Slow instabilities in tropical ocean basin-global atmosphere models. J. Atmos. Sci., 45, 830-852.

Latif, M., T. P. Barnett, M. A. Cane, M. Flugel, N. E. Graham, H von Storch, J.-S. Xu, and S. E. Zebiak, 1994: A review of ENSO prediction studies. Climate Dyn., 9, 167-179.

Lorenz, E. N., 1965: A study of the predictability of a 28-variable atmospheric model. Tellus, 17, 321-333.

Mantua, N. J., and D. S. Battisti, 1994: Evidence for the delayed oscillator mechanism for ENSO: The "observed" oceanic Kelvin mode in the far western Pacific. J. Phys. Oceanogr., 24, $691-$ 699.

_ and $\longrightarrow$ 1995: Aperiodic variability in the Zebiak-Cane coupled ocean-atmosphere model: Ocean-atmosphere interactions in the western equatorial Pacific. J. Climate, 8, 2897-2927,

Molteni, F., R. Buizza, T. N. Palmer, and T. Petroliagis, 1996: The ECMWF ensemble prediction system: Methodology and validation. Quart. J. Roy. Meteor. Soc., 122, 73-119.

Moore, A. M., and R. Kleeman, 1996: The dynamics of error growth and predictability in a coupled model of ENSO. Quart. J. Roy Meteor. Soc., 122, 1405-1446.

Moura, A. D., 1994: Prospects for seasonal-to-interannual climate prediction and applications for sustainable development. World Meteor. Soc. Bull., 43, 207-215.

Palmer, T. N., R. Buizza, E. Molteni, Y.-Q. Chen, and S. Corti, 1994:
Singular vectors and the predictability of weather and climate. Philos. Trans. Roy. Soc. London, 348, 459-475.

Penland, C., and P. D. Sardeshmukh, 1995: The optimal growth of tropical sea surface temperature anomalies. J. Climate, 8, 19992024.

Philander, S. G. H., and R. C. Pacanowski, 1981: The ocean response to cross-equatorial winds (with application to coastal upwelling in low latitudes). Tellus, 33, 201-210.

Rasmusson, E. M., and T. H. Carpenter, 1982: Variations in tropical sea surface temperature and surface wind fields associated with the Southern Oscillation/El Niño. Mon. Wea. Rev., 110, 354384.

Ropelewski, C. F., and M. S. Halpert, 1987: Global and regional scale precipitation patterns associated with E1 Niño/Southern Oscillation. Mon. Wea. Rev., 115, 1606-1626.

Rosati, A., K. Miyakoda, and R. Gudgel, 1997: The impact of ocean initial conditions on ENSO forecasting with a coupled model, Mon. Wea. Rev., 125, 754-772.

Seager, R. S. E. Zebiak, and M. A. Cane, 1988: A model of the tropical Pacific sea surface temperature climatology. J. Geophys. Res., 93, 1265-1280.

Strang, G., 1988: Linear Algebra and its Applications. 3d ed. Academic Press, 442-452.

Thompson, C. J., 1997: Initial conditions for optimal growth in a coupled ocean-atmosphere model of ENSO. J. Atmos. Sci., in press.

Trenberth, K. E., 1991: General characteristics of El Niño-Southern Oscillation. Teleconnections Linking Worldwide Climate Anomalies, M. H. Glantz, R. W. Katz, and N. Nicholls, Eds., Cambridge University Press, 13-42.

Zebiak, S. E., 1986: Atmospheric convergence feedback in a simple model for E1 Niño. Mon. Wea. Rev., 114, 1263-1271.

- and M. A. Cane, 1985: A theory for El Niño and the Southern Oscillation. Science, 228, 1085-1087.

—, and 1987: A model El Niño/Southern Oscillation. Mon. Wea. Rev., 115, 2262-2278. 